\title{
Nucleosynthesis in the Stellar Systems $\omega$ Centauri and M22
}

\author{
G. S. Da Costa $^{\mathrm{A}, \mathrm{C}}$ and A. F. Marino ${ }^{\mathrm{B}}$

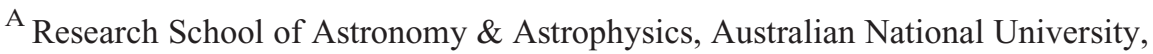 \\ Mt Stromlo Observatory, via Cotter Rd, Weston, ACT 2611, Australia \\ ${ }^{\mathrm{B}}$ Dipartimento di Astronomia, Università di Padova, vicolo dell'Osservatorio 2, \\ I-35122 Padova, Italy \\ ${ }^{\mathrm{C}}$ Corresponding author. Email: gdc@mso.anu.edu.au
}

Received 2010 August 3, accepted 2010 September 7

\begin{abstract}
The stellar system $\omega$ Centauri $(\omega$ Cen) is well known for the large range in elemental abundances among its member stars. Recent work has indicated that the globular cluster M22 (NGC 6656) also possesses an internal abundance range, albeit substantially smaller than that in $\omega$ Cen. Here we compare, as a function of $[\mathrm{Fe} / \mathrm{H}]$, element-to-iron ratios in the two systems for a number of different elements using data from abundance analyses of red giant branch stars. It appears that the nucleosynthetic enrichment processes were very similar in these two systems despite the substantial difference in total mass.
\end{abstract}

Keywords: globular clusters: general — globular clusters: individual (M22, $\omega$ Cen) — stars: abundances

\section{Introduction}

The stellar system $\omega$ Centauri ( $\omega$ Cen) has been known for a long time to be different from most other Galactic globular clusters because its member stars exhibit a wide range in the abundance of heavier elements such as iron and calcium. This contrasts with the situation in most other globular clusters where the size of any intrinsic spread in the abundance of such elements is smaller than the measurement uncertainties (e.g., Kraft \& Ivans 2003; Carretta et al. 2009b). The wide range in heavy-element abundance ${ }^{1}$ in $\omega$ Cen was first demonstrated by abundance measures from spectra of cluster RR Lyrae variables (Freeman \& Rodgers 1975). This then led to the recognition that the large colour width of the red giant branch in the colour-magnitude diagram (CMD) of the cluster, discovered by Woolley (1966) and Geyer (1967) and confirmed by Cannon \& Stobie (1973), was a natural consequence of the heavy-element abundance spread. There now exists a large body of spectroscopic data that characterises the range in $[\mathrm{Fe} / \mathrm{H}]$ in $\omega \mathrm{Cen}$, and more particularly, reveals the complex variations in [element/ $\mathrm{Fe}]$ ratios with $[\mathrm{Fe} / \mathrm{H}]$. The element ratios reveal the nucleosynthetic history of the stellar system, and contributions from both Type II and Type Ia supernovae are recognisable, as is a significant role for asymptotic giant branch (AGB) stars in the chemical evolution.

The difference between $\omega$ Cen and other globular clusters has led to the suggestion that $\omega$ Cen may have formed in a different way from the other clusters - that it is the nuclear remnant of a former dwarf galaxy that has been

\footnotetext{
$\overline{{ }^{1} \text { Usually represented by }[\mathrm{Fe} / \mathrm{H}] \text {, the logarithm of the iron abundance }}$ relative to that found in the Sun.
}

tidally disrupted by the Milky Way (e.g., Freeman 1993). Bekki \& Freeman (2003), for example, have shown that this is dynamically plausible. The different evolutionary environment may then have allowed additional chemical processes that do not occur in 'regular' globular clusters (e. g., Bekki \& Norris 2006; Romano et al. 2007, 2010).

The stellar system $\omega$ Cen is, however, no longer the only cluster in which a definite range in heavy-element abundance is present among the member stars. For example, following the initial photometric studies of Sarajedini \& Layden (1995), Bellazzini et al. (2008) have used spectra at the Ca II triplet of a large sample of member stars to show that the globular cluster M54, which is located at the centre of the Sagittarius dwarf galaxy, possesses an internal abundance range characterised by $\sigma([\mathrm{Fe} / \mathrm{H}])_{\mathrm{int}} \approx 0.14 \mathrm{dex}$ (see also the very recent work of Carretta et al. 2010c). Further, two other globular clusters have recently been shown to have internal ranges in heavy-element abundance. The first of these is the Galactic bulge globular cluster Terzan 5, for which observations presented in Ferraro et al. (2009) reveal the presence of two distinct populations that differ by a factor of $\sim 3$ in $[\mathrm{Fe} / \mathrm{H}]$, and which likely also differ significantly in age, with the more metal-rich population being younger (Ferraro et al. 2009). The second globular cluster is M22 (NGC 6656), a stellar system long suspected of possessing similar properties to $\omega$ Cen (e.g., Norris \& Freeman 1983), despite the fact that with $\mathrm{M}_{\mathrm{V}} \approx$ -8.5 , it is considerably less luminous than either $\omega$ Cen or M54, which have $\mathrm{M}_{\mathrm{V}} \approx-10.3$ and -10.0 , respectively (Harris 1996). For M22, Da Costa et al. (2009) used spectra at the $\mathrm{Ca}$ II triplet of $41 \mathrm{red}$ giants to derive an $[\mathrm{Fe} / \mathrm{H}]$ abundance distribution whose significant width could not be explained by differential reddening effects. The 
inter-quartile range for the observed abundances is $0.24 \mathrm{dex}$, and the distribution suggests the presence of at least two components, whose mean metallicities differ by $0.26 \mathrm{dex}$ (Da Costa et al. 2009). An intrinsic variation in star-to-star abundances in M22 was also revealed recently by the highresolution spectroscopic study of 17 M22 red giants presented in Marino et al. (2009). These authors found not only that there is an intrinsic range in $[\mathrm{Fe} / \mathrm{H}]$ of order $0.15 \mathrm{dex}$, but also that two groups of stars are present in the cluster, with the iron-richer stars having higher calcium and $s$ process element abundances relative to the iron-poorer stars (Marino et al. 2009).

While the number of clusters showing internal heavyelement abundance variations is small, this is not the case for the lighter elements. After many decades of work, the light elements $\mathrm{C}, \mathrm{N}, \mathrm{O}, \mathrm{Na}, \mathrm{Al}$, and $\mathrm{Mg}$ are now known to vary significantly and systematically within all globular clusters for which adequate data are available (e.g., Gratton et al. 2004; Carretta et al. 2010a). The sense of the variations is such that increases in the nitrogen, sodium and aluminium abundances are coupled with decreases in the carbon, oxygen and magnesium abundances. Together the effects are referred to as the $\mathrm{O}-\mathrm{Na}$ anti-correlation. The abundance patterns seen in the Na-rich, O-poor stars are consistent with being produced by the operation of the $\mathrm{CNO}$ cycle, together with proton-capture reactions on $\mathrm{Ne}$ and $\mathrm{Mg}$ seeds during H-burning at high temperatures (Denisenkov \& Denisenkova 1990; Langer et al. 1993). However, the actual site of the nucleosynthesis is not yet clearly established, with the most likely candidates being intermediate-mass AGB stars (e.g., D'Antona \& Ventura 2007) or rapidly rotating massive stars (e.g., Decressin et al. 2007). Nevertheless, given that the 'anomalies' are found among main sequence stars in at least some clusters, the origin of the abundance patterns must be related to a process or processes that occurred during the formation of the clusters. In this respect $\omega$ Cen and M 22 are no different from other globular clusters - the O-Na anticorrelation is clearly present in both systems (Norris \& Da Costa 1995a; Marino et al. 2009).

The stellar system $\omega$ Cen has one further property that distinguishes it from most other globular clusters: the likely presence of a large range in helium abundance. This property is inferred from the presence of a double main sequence in a high-precision Hubble Space Telescopebased CMD for the cluster (Bedin et al. 2004, see also Bellini et al. (2010)). In this CMD the bluer sequence is less numerous and of higher abundance than the redder sequence (Piotto et al. 2005). The observations are best interpreted as indicating that the stars in the bluer sequence are enhanced in helium by $\Delta \mathrm{Y} \approx 0.10-0.15$ relative to those in the redder sequence (Piotto et al. 2005). Such large He abundance ranges have also been suggested to occur in a small number of other luminous, massive globular clusters. The prime example is NGC 2808, whose CMD shows a triple main sequence (Piotto et al. 2007). Given the lack of heavy-element abundance variation in the cluster (e.g., Carretta et al.
$2009 b$ ), the main sequence structure is best interpreted as indicating distinct $\mathrm{He}$ abundance groups, which are then likely also related to the multi-modal structure of the horizontal branch in the cluster CMD (e.g., D'Antona et al. 2005). Other clusters for which very precise CMDs reveal the existence of main sequence colour widths in excess of that expected from the photometric errors include 47 Tuc (Anderson et al. 2009) and NGC 6752 (Milone et al. 2010). The origin of the postulated large $\mathrm{Y}$ variations in these clusters is poorly understood though the same candidates as those employed for explaining the O-Na anti-correlation are often invoked (e.g., Renzini 2008). However, although some structure is present in the vicinity of the subgiant branch in an HST-based CMD for M22 (Piotto 2009), there is no evidence for any substantial He abundance variations within this cluster. In this respect then, M22 clearly differs from $\omega$ Cen.

In this paper we concentrate on a comparison of the element-to-iron abundance ratios, as a function of $[\mathrm{Fe} / \mathrm{H}]$, of the red giants in $\omega$ Cen and M22. This allows an investigation of the extent to which similar nucleosynthetic activities occurred during their evolution, which in turn, can constrain their origin. The observational data used for the comparison are outlined in the next section. The comparison is carried out in Section 3, and the results are discussed in Section 4. Briefly, a considerable degree of similarity is found between the two systems, suggesting that they underwent analogous evolutionary processes.

\section{Observational Data}

There are a number of abundance analyses based on highresolution spectroscopy available for $\omega$ Cen red giants. These include Norris \& Da Costa (1995b), Smith et al. (2000), Pancino et al. (2002) and Johnson et al. (2009), each of which differ in sample size, elements studied, and metallicity range covered. We will use primarily the results of Norris \& Da Costa (1995b), who give abundances for a large number of elements derived from observations of a sample of 40 red giants chosen to cover (almost) the full range of $[\mathrm{Fe} / \mathrm{H}]$ values exhibited by $\omega$ Cen stars. Where necessary we will also draw on the results of Smith et al. (2000) and Johnson et al. (2009): both these papers have demonstrated that any systematic differences between their results and those of Norris \& Da Costa (1995b) are small, and have their origin in different choices of $g f$-values, lines measured, and atmospheric parameters. ${ }^{2}$

In contrast to $\omega \mathrm{Cen}$, the only published highdispersion study of a large sample of M22 red giants is that of Marino et al. (2009). These authors analysed highresolution VLT/UVES observations of 17 M22 red giants

\footnotetext{
${ }^{2}$ After this manuscript was submitted, a paper by Johnson \& Pilachowski (2010) appeared on the arXiv preprint archive. The paper provides $\mathrm{O}, \mathrm{Na}, \mathrm{Al}, \mathrm{Si}, \mathrm{Ca}, \mathrm{Sc}, \mathrm{Ti}, \mathrm{Fe}, \mathrm{Ni}, \mathrm{La}$ and $\mathrm{Eu}$ abundances for a very large sample of $855 \omega$ Cen red giants. We have attempted to include results from that paper where appropriate, but note that Johnson \& Pilachowski (2010) show that any systematic offsets between their abundances and those of Norris \& Da Costa (1995b) are also small.
} 


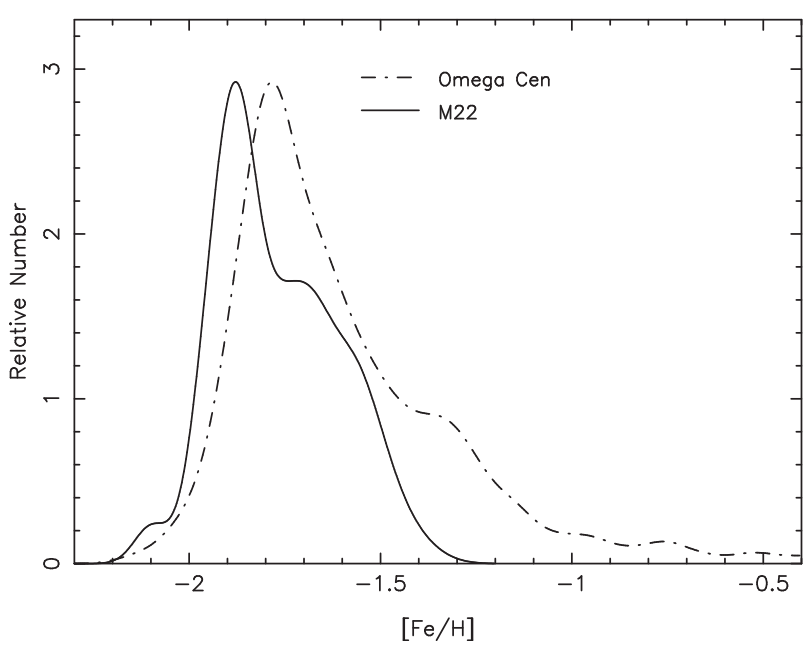

Figure 1 Generalised histograms showing the abundance distributions of $\omega$ Cen (dot-dash line) and M22 (solid line). The two distributions have been normalised to the same peak height. See text for details.

as well as somewhat lower-resolution VLT/GIRAFFE data for a further 14 red giants, one of which is in common with the UVES sample.

\section{Comparing $\omega$ Centauri and M22 Element-to-Iron Abundance Ratios}

To set the scene we show in Figure 1 generalised histograms for the abundance distributions in M22 and $\omega$ Cen. The M22 data are from Da Costa et al. (2009) while the $[\mathrm{Fe} / \mathrm{H}]$ distribution for $\omega$ Cen is derived from the $[\mathrm{Ca} / \mathrm{H}]$ distribution presented in Norris et al. (1996b), by assuming a constant $[\mathrm{Ca} / \mathrm{Fe}]$ ratio of $0.4 \mathrm{dex}$ (Norris \& Da Costa 1995b, see also Figure 2). The two distributions, which incorporate similar abundance uncertainties (see Da Costa et al. 2009) have been scaled to have the same peak height. As noted by Da Costa et al. (2009), the distributions show a considerable degree of similarity. Both rise rapidly on the metal-poor side to a well-defined peak, with that for M22 being $\sim 0.09$ dex more metal-poor than that for $\omega$ Cen. Both distributions then decrease with increasing metallicity and there are clear hints of the presence of multiple populations. The $\omega$ Cen distribution continues to substantially higher abundances than that for $\mathrm{M} 22$, a result which is most likely not due solely to the smaller sample size for M22 (Da Costa et al. 2009).

We now turn to a comparison of element-to-iron abundance ratios between these two stellar systems, starting first with the $\alpha$ - and iron-peak elements, which constrain the contributions from Type II and Type Ia supernovae. This is followed by the elements $\mathrm{O}, \mathrm{Na}$, and $\mathrm{Al}$, which are involved in the poorly understood globularcluster-specific nucleosynthesis process, and finally by the $r$-and $s$-process elements, the latter of which is closely tied to nucleosynthetic contributions from AGB stars.

\subsection{The $\alpha$ - and Iron-Peak Elements}

In Figure 2 we show element-to-iron ratios as a function of $[\mathrm{Fe} / \mathrm{H}]$ for the $\alpha$-elements $\mathrm{Si}$ and $\mathrm{Ca}$. We note that the

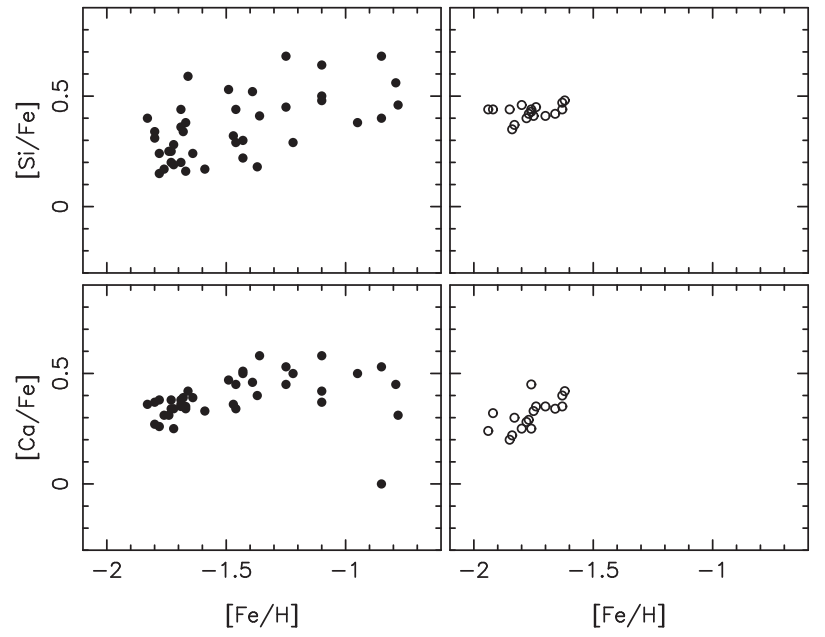

Figure 2 Element-to-iron abundance ratios for the $\alpha$-elements silicon (upper panels) and calcium (lower panels) as a function of $[\mathrm{Fe} / \mathrm{H}]$ for $\omega$ Cen red giants (left panels) and for M22 red giants (right panels). Unless otherwise noted, in this and subsequent figures the $\omega$ Cen data are from Norris \& Da Costa (1995b) and the M22 data are from Marino et al. (2009).

$[\mathrm{Si} / \mathrm{Fe}]$ ratio for the $\omega$ Cen stars shows a sizeable scatter with an indication of a possible trend of increasing $[\mathrm{Si} / \mathrm{Fe}]$ with $[\mathrm{Fe} / \mathrm{H}]$. The $[\mathrm{Si} / \mathrm{Fe}]$ data for the relatively small number of red giants studied in Smith et al. (2000) show no such trend, but the large sample of stars studied in Johnson \& Pilachowski (2010) reveals that the behaviour of $[\mathrm{Si} / \mathrm{Fe}]$ with $[\mathrm{Fe} / \mathrm{H}]$ in $\omega \mathrm{Cen}$ is quite complex. The bulk of the population has $[\mathrm{Si} / \mathrm{Fe}]=0.29$ but the ratio rises to $[\mathrm{Si} / \mathrm{Fe}]=0.45$ for the most metal-rich stars, consistent with the Norris \& Da Costa (1995b) data shown in the figure. Similarly, the Norris \& Da Costa (1995b) [Ca/Fe] data plotted in the lower left panel of Figure 2 show some indication of a slight increase in $[\mathrm{Ca} / \mathrm{Fe}]$ with $[\mathrm{Fe} / \mathrm{H}]$ for the stars more metal-poor than $[\mathrm{Fe} / \mathrm{H}] \approx-1.3 \mathrm{dex}$. The large-sample study of Johnson \& Pilachowski (2010) reveals similar behaviour, in that while the bulk of the population has $[\mathrm{Ca} / \mathrm{Fe}]=0.26$, there is a $\operatorname{small}(\sim 0.1 \mathrm{dex})$ increase in the abundance ratio as $[\mathrm{Fe} / \mathrm{H}]$ rises. However, the stars with $[\mathrm{Fe} / \mathrm{H}]>-1$ possess similar $[\mathrm{Ca} / \mathrm{Fe}]$ values to that for the bulk of the population (Johnson \& Pilachowski 2010). On the other hand, for M22 there is clearly no trend in $[\mathrm{Si} / \mathrm{Fe}]$ with $[\mathrm{Fe} / \mathrm{H}]$, and the dispersion in the $[\mathrm{Si} / \mathrm{Fe}]$ values is particularly small. However, for $[\mathrm{Ca} / \mathrm{Fe}]$, there may also be a small increase in $[\mathrm{Ca} / \mathrm{Fe}]$ with $[\mathrm{Fe} / \mathrm{H}]$ similar to that seen for $\omega \mathrm{Cen}$. Further investigation is needed to confirm the existence of this possible trend.

Similar plots for the other $\alpha$-elements for which abundances are available, $\mathrm{Mg}$ and $\mathrm{Ti}$, show the same result - the red giants in both clusters have essentially constant and positive $[\mathrm{Mg} / \mathrm{Fe}]$ and $[\mathrm{Ti} / \mathrm{Fe}]$ values regardless of $[\mathrm{Fe} / \mathrm{H}] .^{3}$ The one exception, as noted in Norris \& Da Costa (1995b), is that the $\omega$ Cen sample shows a small number of $\mathrm{Mg}$-depleted stars, with $[\mathrm{Mg} / \mathrm{Fe}]$ values in the

\footnotetext{
${ }^{3}$ The extensive sample of Johnson \& Pilachowski (2010) shows that $[\mathrm{Ti} / \mathrm{Fe}]$ behaves similarly to $[\mathrm{Si} / \mathrm{Fe}]$ and $[\mathrm{Ca} / \mathrm{Fe}]$ in that there is a slight increase in $[\mathrm{Ti} / \mathrm{Fe}]$ with increasing $[\mathrm{Fe} / \mathrm{H}]$.
} 


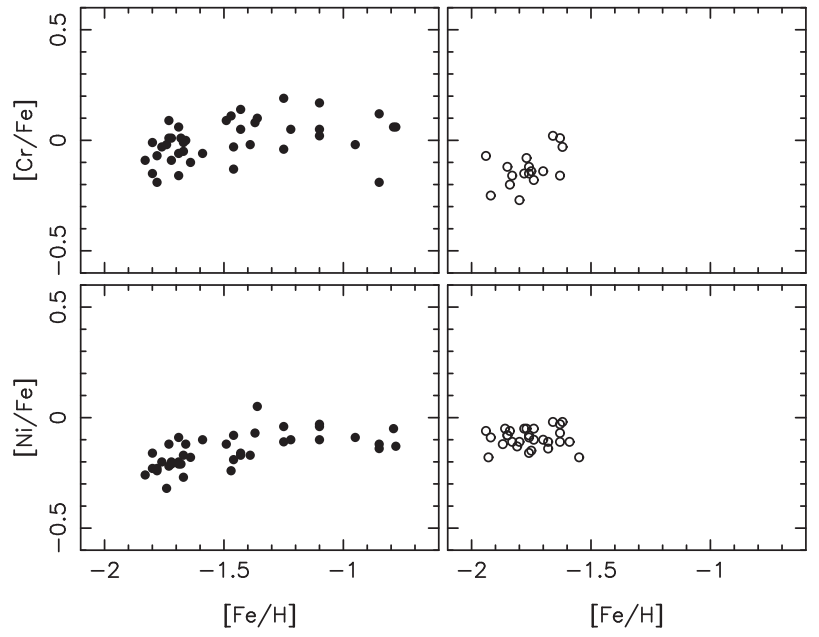

Figure 3 Element-to-iron abundance ratios for the iron-peak elements chromium (upper panels) and nickel (lower panels) as a function of $[\mathrm{Fe} / \mathrm{H}]$ for $\omega$ Cen red giants (left panels) and for M22 red giants (right panels).

range $\sim 0.05$ to -0.2 dex. These stars, however, are all strongly enhanced in $[\mathrm{Al} / \mathrm{Fe}]$ (Norris \& Da Costa 1995b) presumably reflecting a significant contribution to the gas from which they formed from material processed through the $\mathrm{Mg}$-Al cycle. No such Mg-depleted stars are found in the current M22 sample (Marino et al. 2009).

Consequently, in general it appears that both $\omega$ Cen and M22 show a similar degree of enrichment from Type II supernovae, with the value of $[\alpha / \mathrm{Fe}]$ also being closely similar to that for Galactic halo field stars at comparable [Fe/H] values (e.g., Gratton et al. 2004). However, we note in passing that according to Pancino et al. (2002), at the highest $[\mathrm{Fe} / \mathrm{H}]$ values (not shown in Figure 2), the red giants in $\omega$ Cen have lower $[\alpha / \mathrm{Fe}]$ values. In particular, Pancino et al. (2002) have analyzed three $\omega$ Cen red giants with $[\mathrm{Fe} / \mathrm{H}] \approx-0.6$, finding $[\alpha / \mathrm{Fe}]=0.10 \pm 0.04(\alpha=\mathrm{Ca}$, $\mathrm{Si}$ ) compared to three red giants at $[\mathrm{Fe} / \mathrm{H}] \approx-1.0$ for which $[\alpha / \mathrm{Fe}]=0.29 \pm 0.01$, a value comparable to those seen in Figure 2. The lower $[\alpha / \mathrm{Fe}]$ value at larger $[\mathrm{Fe} / \mathrm{H}]$ is taken as an indication that Type Ia supernovae have contributed to the chemical evolution of $\omega$ Cen. However, Johnson \& Pilachowski (2010) have also analysed two of the three metal-rich stars studied in Pancino et al. (2002), and find higher $[\mathrm{Si} / \mathrm{Fe}]$ and $[\mathrm{Ca} / \mathrm{Fe}]$ abundance ratios. Whether this is solely the result of differences in adopted $g f$-values, model atmospheres and lines measured (Johnson \& Pilachowski 2010), or is a more fundamental discrepancy, is unclear. The M22 sample lacks any low- $[\alpha / \mathrm{Fe}]$ stars, though this is perhaps not surprising if one supposes that the smaller total abundance range in M22 means that the duration of the chemical evolution epoch was shorter in that cluster than for $\omega$ Cen.

In Figure 3 we show $[\mathrm{Cr} / \mathrm{Fe}]$ and $[\mathrm{Ni} / \mathrm{Fe}]$ as a function of $[\mathrm{Fe} / \mathrm{H}]$ for the $\omega$ Cen and M22 red giant samples. Plots of the abundance ratios for scandium and vanadium, the other iron-peak elements for which data are available, show similar behaviour - no dependence on $[\mathrm{Fe} / \mathrm{H}]$ and ratio values near solar — and the

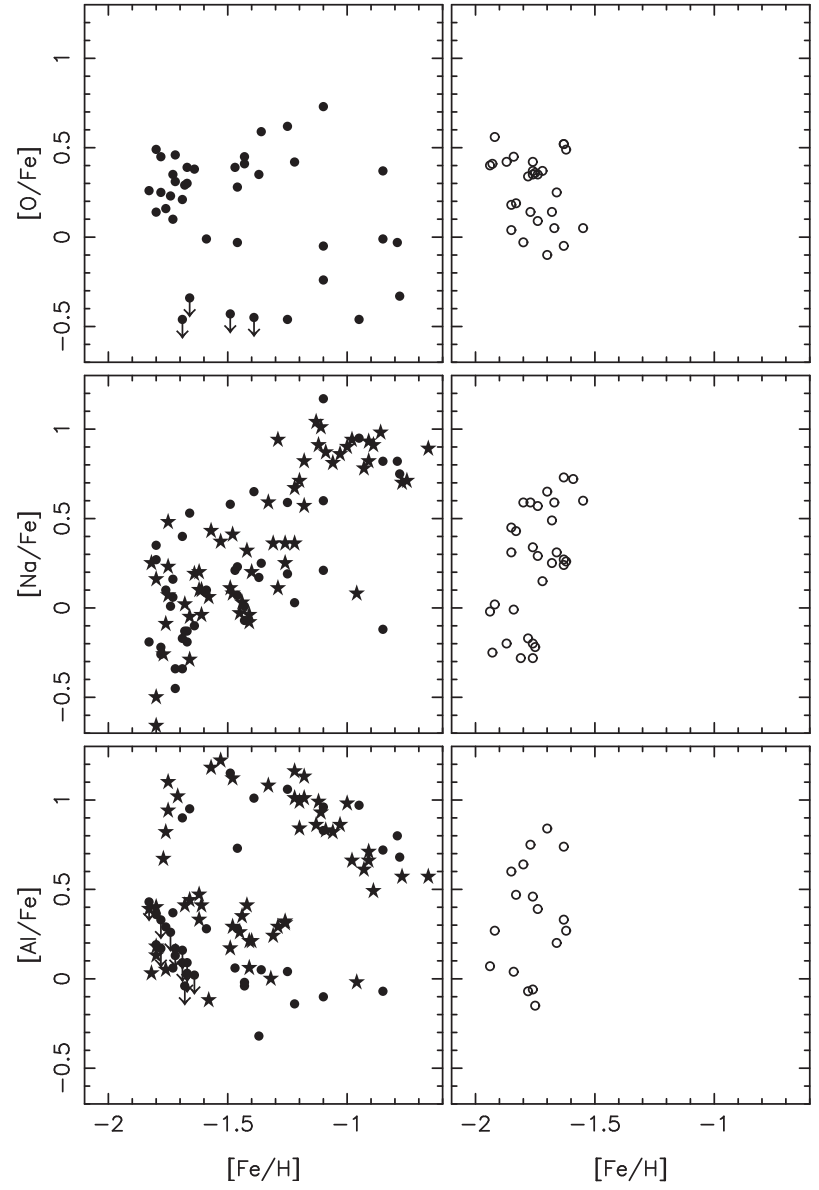

Figure 4 Element-to-iron abundance ratios for the elements oxygen (upper panels), sodium (middle panels) and aluminium (lower panels) as a function of $[\mathrm{Fe} / \mathrm{H}]$ for $\omega$ Cen red giants (left panels) and for M22 red giants (right panels). For $\omega$ Cen, the filled circles are from Norris \& Da Costa (1995b) while the filled stars are from Johnson et al. (2009). $\omega$ Cen points with downward arrows represent upper limits.

extensive data of Johnson \& Pilachowski (2010) for $[\mathrm{Ni} / \mathrm{Fe}]$ and $[\mathrm{Sc} / \mathrm{Fe}]$ show the same result. Further, as for the $\alpha$-elements, the abundance ratios for the $\omega$ Cen and M22 red giants are similar to those for halo field stars at similar $[\mathrm{Fe} / \mathrm{H}]$ values (e.g., Gratton et al. 2004). The similarity between the two stellar systems and the halo field is not surprising since at these metallicities the production of iron-peak elements like $\mathrm{Cr}$ and $\mathrm{Ni}$ is expected to closely follow that of Fe.

\subsection{Oxygen, Sodium and Aluminium}

In Figure 4 we show the oxygen, sodium and aluminium abundance ratios with respect to iron for $\omega$ Cen (left panels) and M22 (right panels). The $\omega$ Cen data for $[\mathrm{O} / \mathrm{Fe}]$ come from Norris \& Da Costa (1995b) while that for $[\mathrm{Na} / \mathrm{Fe}]$ and $[\mathrm{Al} / \mathrm{Fe}]$ come from both Norris \& Da Costa (1995b) and Johnson et al. (2009). There are 7 stars in common. For these 7 stars, the mean difference in $[\mathrm{Na} / \mathrm{Fe}]$, in the sense of Johnson et al. (2009) minus Norris \& Da Costa (1995b), is -0.11 dex with a sigma of $0.20 \mathrm{dex}$. For $[\mathrm{Al} / \mathrm{Fe}]$, Norris \& Da Costa (1995a) give only upper limits for two of the 
common stars, but for the remaining five the mean difference in $[\mathrm{Al} / \mathrm{Fe}]$ is +0.08 dex with a sigma of 0.14 dex. Thus any systematic difference between the two sets of abundance determinations is minor, and can be neglected given the substantial range in $[\mathrm{Na} / \mathrm{Fe}]$ and $[\mathrm{Al} / \mathrm{Fe}]$ observed. The more extensive $\omega$ Cen data set of Johnson \& Pilachowski (2010) shows essentially the same structure as seen in the left panels of Figure 4.

Turning first to oxygen, we see that the upper limit for the $[\mathrm{O} / \mathrm{Fe}]$ values, namely $[\mathrm{O} / \mathrm{Fe}] \approx 0.4-0.5$, is similar in both clusters and shows no obvious trend with $[\mathrm{Fe} / \mathrm{H}]$. This is not surprising since the $[\mathrm{O} / \mathrm{Fe}]$ ratio for the 'O-rich' stars is expected to be similar to the element-to-iron abundance ratio for other $\alpha$-elements (e.g., Figure 2). Nevertheless, as is common among globular-cluster stars, both $\omega$ Cen and M22 also exhibit populations of red giants with depleted $[\mathrm{O} / \mathrm{Fe}]$ ratios, which correlate with enhanced $\mathrm{Na}$ and $\mathrm{Al}$ abundances (e.g., Norris \& Da Costa 1995a; Marino et al. 2009). Specifically for the [O/Fe] ratios, the only noteworthy difference between the two stellar systems, within the range of $[\mathrm{Fe} / \mathrm{H}]$ overlap, is the presence in $\omega$ Cen of a small number of stars showing very low $[\mathrm{O} / \mathrm{Fe}]$ values; such stars are not seen in the M22 sample of Marino et al. (2009). Further, it is also evident from the upper left panel of Figure 4 that the relative frequency of O-depleted stars in $\omega$ Cen is larger for $[\mathrm{Fe} / \mathrm{H}] \geq-1.2$, compared to the bulk of the population at $[\mathrm{Fe} / \mathrm{H}] \approx-1.75 \mathrm{dex}$. Such an effect has already been noted by, for example, Norris \& Da Costa (1995b) and Carretta et al. (2010b).

As regards $[\mathrm{Na} / \mathrm{Fe}]$, again within the $[\mathrm{Fe} / \mathrm{H}]$ interval common to both $\omega$ Cen and $\mathrm{M} 22$, the range in $[\mathrm{Na} / \mathrm{Fe}]$ seen in both systems is comparable, of order $0.8-1$ dex. This $[\mathrm{Na} / \mathrm{Fe}]$ range is in agreement with that seen in most globular clusters (e.g., Carretta et al. 2010a). Moreover, the lower $[\mathrm{Na} / \mathrm{Fe}]$ values, which correspond to the O-rich stars, are similar between the two objects as well as being similar to the $[\mathrm{Na} / \mathrm{Fe}]$ values for halo field stars of comparable [Fe/H] (e.g., Johnson et al. 2009; Carretta et al. 2010a). However, as noted by Johnson et al. (2009) and Carretta et al. (2010b), at $[\mathrm{Fe} / \mathrm{H}] \geq-1.2$, stars with notably larger enhancements in $[\mathrm{Na} / \mathrm{Fe}]$ dominate in $\omega$ Cen, and there are relatively few stars with low values of $[\mathrm{Na} / \mathrm{Fe}]$. In other words, using the terminology of Carretta et al. (2009a), the metal-rich population of $\omega$ Cen is dominated by 'extreme' stars while 'primordial' stars are rare at these metallicities (see also Johnson et al. 2009; Johnson \& Pilachowski 2010). In contrast, M22, at least as regards the sample of Marino et al. (2009), lacks such 'extreme' stars.

For $[\mathrm{Al} / \mathrm{Fe}]$, if we again restrict ourselves to the $[\mathrm{Fe} / \mathrm{H}]$ interval in which $\omega$ Cen and M22 have stars in common, we see results that are broadly similar to those for $[\mathrm{Na} / \mathrm{Fe}]$. In particular, a range in $[\mathrm{Al} / \mathrm{Fe}]$ of $\sim 1 \mathrm{dex}$ is present in both systems though there is some indication that the M22 stars have a slightly lower 'primordial' [A1/Fe] and a lower 'enhanced' $[\mathrm{Al} / \mathrm{Fe}]$ than do the $\omega$ Cen stars. This may be a result of different choices of analysis parameters rather than any real offset. The combined Norris \& Da Costa (1995b) and Johnson et al. (2009) results for $\omega$ Cen in the bottom left panel of Figure 4 present an intriguing picture. For $[\mathrm{Fe} / \mathrm{H}]$ values below approximately $-1.2 \mathrm{dex}$, it appears that the maximum $[\mathrm{Al} / \mathrm{Fe}]$ value is constant at $\sim 1.0$ dex, but at higher abundances, it decreases with increasing $[\mathrm{Fe} / \mathrm{H}]$. This effect contrasts with the situation for $[\mathrm{Na} / \mathrm{Fe}]$ in $\omega$ Cen, where the highest values of $[\mathrm{Na} / \mathrm{Fe}]$ occur at and above $[\mathrm{Fe} / \mathrm{H}] \approx-1.2$ dex. Johnson et al. (2009) discuss the possible implications of this result, noting that it is certainly consistent with current AGB nucleosynthesis models, which predict that more Al is produced at low metallicity, and more $\mathrm{Na}$ at higher metallicity, due to lower temperatures at the bottom of the convective envelope, and shallower mixing, in more metal-rich stars (e.g., Ventura \& D’Antona 2008).

\section{3 r- and s-Process Elements}

We first examine the possible contribution of $r$-process nucleosynthesis in $\omega$ Cen and M22 by investigating the element-to-iron abundance ratio for europium, an element whose abundance is mainly determined by $r$-process nucleosynthesis. The observational results are shown in the left panels of Figure 5 where the $\omega$ Cen data have been taken from Johnson et al. (2009) as the Norris \& Da Costa
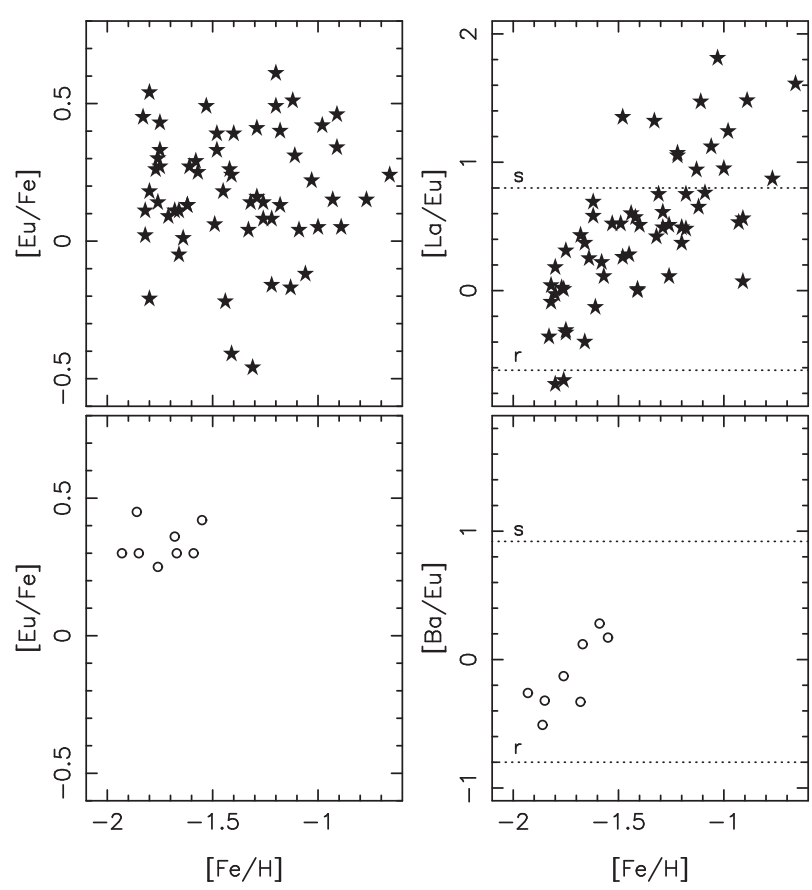

Figure 5 Left panels. Europium-to-iron ratios as a function of $[\mathrm{Fe} / \mathrm{H}]$ for red giants in $\omega$ Cen (upper panel) and in M22 (lower panel). Note the absence of any clear trend of $[\mathrm{Eu} / \mathrm{Fe}]$ with $[\mathrm{Fe} / \mathrm{H}]$ in both systems. Right panels. Lanthanum-to-europium ratios as a function of $[\mathrm{Fe} / \mathrm{H}]$ for $\omega$ Cen red giants (upper panel) and barium-toeuropium ratios as a function of $[\mathrm{Fe} / \mathrm{H}]$ for M22 stars (lower panel). The dotted lines in the right panels indicate the solar system $r$-process ratio and an extreme $s$-process ratio taken from McWilliam (1997). The increase of the ratios with $[\mathrm{Fe} / \mathrm{H}]$ indicates the dominance of $s$ process contributions to the nucleosynthesis. The $\omega \mathrm{Cen}$ data are from Johnson et al. (2009). 
(1995b) data for Eu contains only upper limits for a limited sub-sample of stars. Again the recent results of Johnson \& Pilachowski (2010) are similar to those shown. The vast majority of Galactic globular clusters have $[\mathrm{Eu} / \mathrm{Fe}]=+0.40$ dex with relatively little cluster-tocluster scatter (e.g., Gratton et al. 2004), a value consistent with halo field stars of comparable $[\mathrm{Fe} / \mathrm{H}]$ (Gratton et al. 2004). The value for the M22 stars in the lower left panel is clearly consistent with this value and there is no indication of any intrinsic variation in the $[\mathrm{Eu} / \mathrm{Fe}]$ values. The $\omega$ Cen values shown in the upper left panel exhibit an apparently substantial scatter, though Johnson et al. (2009) do not comment on it, presumably indicating that the scatter is primarily due to observational errors. Certainly the small number of $\omega$ Cen stars with apparently low $[\mathrm{Eu} / \mathrm{Fe}]$ ratios are not discrepant in the $[\mathrm{La} / \mathrm{Eu}]$ plot shown in the upper right panel of Figure 5. Specifically, only two of the seven stars with $[\mathrm{Eu} / \mathrm{Fe}] \leq-0.1$ in the upper left panel of Figure 5 also have $[\mathrm{La} / \mathrm{Eu}] \geq 1.0$ in the upper right panel of the figure, while the other eight stars with $[\mathrm{La} / \mathrm{Eu}] \geq 1.0$ all have $[\mathrm{Eu} / \mathrm{Fe}]$ values consistent with the bulk of the $\omega$ Cen sample.

Johnson et al. (2009) and Johnson \& Pilachowski (2010) note that the mean $[\mathrm{Eu} / \mathrm{Fe}]$ for the $\omega$ Cen stars is 0.1 to 0.2 dex lower than the value for other globular clusters, including M22, and for the halo field. Nevertheless, the lack of any variation of $[\mathrm{Eu} / \mathrm{Fe}]$ with $[\mathrm{Fe} / \mathrm{H}]$ in either $\omega$ Cen or M22 indicates that the $r$-process nucleosynthesis in these systems must be tightly coupled to that of iron. This is in contrast to the situation in the metal-poor globular cluster M15 where a distinct range in $[\mathrm{Eu} / \mathrm{Fe}]$ abundances, of order $0.5 \mathrm{dex}$, is seen at constant $[\mathrm{Fe} / \mathrm{H}]$ (Sneden et al. 1997; Otsuki et al. 2006).

As regards the $s$-process contribution, we show in the upper right panel of Figure 5 the abundance ratio [La/Eu] as a function of $[\mathrm{Fe} / \mathrm{H}]$, again using the data of Johnson et al. (2009). Lanthanum is primarily synthesized by the $s$-process while, as noted above, Eu traces the $r$-process. Their abundance ratio is therefore a measure of the relative importance of these two neutron-capture nucleosynthetic processes (see, e.g., McWilliam 1997). The data reinforce the conclusions of Norris \& Da Costa (1995b), Smith et al. (2000), Johnson et al. (2009) and Johnson \& Pilachowski (2010) that as $[\mathrm{Fe} / \mathrm{H}]$ increases in $\omega$ Cen, the $s$-process dominates the enrichment of the neutroncapture elements.

This appears also to be the case for M22. The lower right panel of Figure 5 shows a similar plot of abundance ratio versus $[\mathrm{Fe} / \mathrm{H}]$ for the $\mathrm{M} 22$ red giants in the sample of Marino et al. (2009). Here we have used barium as the $s$ process tracer since Marino et al. (2009) did not measure La abundances. While the number of M22 stars in the sample with measured abundances for both elements is relatively small, the data nevertheless show a similar trend of increasing abundance ratio with increasing $[\mathrm{Fe} / \mathrm{H}]$. Indeed if we restrict the $\omega$ Cen data to only those stars with $[\mathrm{Fe} / \mathrm{H}] \leq-1.5$, i.e., those which overlap in $[\mathrm{Fe} / \mathrm{H}]$ with the M22 sample, then the slope of the
([La/Eu], $[\mathrm{Fe} / \mathrm{H}])$ relation is essentially identical to the slope of the $([\mathrm{Ba} / \mathrm{Eu}],[\mathrm{Fe} / \mathrm{H}])$ relation for the M22 stars. In other words the rate of increase with $[\mathrm{Fe} / \mathrm{H}]$ of the relative $s$-process contribution appears to have been the same in both stellar systems, again suggesting that similar nucleosynthetic processes are at work.

The high values of $[\mathrm{Ba}, \mathrm{La} / \mathrm{Eu}]$ at larger $[\mathrm{Fe} / \mathrm{H}]$ in both M22 and $\omega$ Cen contrast with the situation in the majority of other globular clusters, where the ratio typically reveals a dominant $r$-process contribution. Gratton et al. (2004) list a mean $[\mathrm{Ba}, \mathrm{La} / \mathrm{Eu}]$ value of $-0.23 \pm 0.04(\sigma=0.21)$ dex for 28 clusters with $[\mathrm{Fe} / \mathrm{H}]$ values between -2.4 and -0.7 dex. The one clear exception is M4, for which Ivans et al. (1999) give $\langle[\mathrm{Ba} / \mathrm{Eu}]\rangle=+0.25$ dex, indicative of a more substantive $s$-process contribution to the gas from which the M4 stars formed. We note, however, that there is no evidence for any intrinsic spread in the barium (or lanthanum) abundances in M4 (Ivans et al. 1999; Marino et al. 2008).

We now look at the $s$-process elements in more detail. In the left panels of Figure 6 we show the element-to-iron ratios for $\omega$ Cen red giants for the $s$-process elements yttrium, barium and neodymium as a function of $[\mathrm{Fe} / \mathrm{H}]$ using data from Norris \& Da Costa (1995b). As is evident from the figure, all three elements show similar behaviour. There is an initial rapid rise in the element-to-iron abundance ratio, from a presumably $r$-process-driven initial value, up to abundance ratios that are significantly above solar. At higher metallicities, however, the increase ceases and the abundance ratio remains constant as the iron abundance continues to rise. The total change in the abundance ratios is of order 0.9 dex in all three cases.

We have endeavoured to quantify this behaviour as follows. First, we adopted $[\mathrm{Fe} / \mathrm{H}]=-1.30$ as the abundance above which the $[\mathrm{Y}, \mathrm{Ba}, \mathrm{Nd} / \mathrm{Fe}]$ abundance ratios are constant. We then used a least-squares method to determine the slope of the relation between the abundance ratio and $[\mathrm{Fe} / \mathrm{H}]$ for the stars less abundant than the adopted limit. In carrying out the least-squares fits, two stars, namely the extremely CN-strong star ROA 144 and the $\mathrm{CH}$ star ROA 279, were excluded. Both these stars have anomalously large $[s$-process $/ \mathrm{Fe}]$ ratios for their $[\mathrm{Fe} /$ $\mathrm{H}$ ] values (Norris \& Da Costa 1995b) and may possess additional $s$-process enhancements resulting from binary mass transfer. ROA 53 was also excluded from the ([Ba/ $\mathrm{Fe}],[\mathrm{Fe} / \mathrm{H}])$ fit because of the apparently high abundance ratio for its $[\mathrm{Fe} / \mathrm{H}]$, but this star is not unusual as regards $[\mathrm{Y} / \mathrm{Fe}]$ or $[\mathrm{Nd} / \mathrm{Fe}]$, nor in $[\mathrm{La} / \mathrm{Fe}]$ for that matter.

The slopes found for each element ratio were all consistent with each other to within the uncertainties. Consequently, the values were averaged and a single slope of $1.70 \mathrm{dex} / \mathrm{dex}$ for $\Delta[s / \mathrm{Fe}] / \Delta[\mathrm{Fe} / \mathrm{H}]$ refitted to each of the element ratio datasets. The value of the element ratio for the fitted relation at $[\mathrm{Fe} / \mathrm{H}]=-1.3$ was then extended to higher metallicities, and it provides a satisfactory representation of the data. We note also that the $([\mathrm{La} / \mathrm{Fe}],[\mathrm{Fe} / \mathrm{H}])$ data of Johnson et al. (2009) are consistent with these results. Excluding the seven stars with 
$[\mathrm{La} / \mathrm{Fe}]$ values exceeding $+1.3 \mathrm{dex}$, which may have had additional $s$-process enhancement from mass transfer in binary star systems, ${ }^{4}$ the distribution of the remaining stars is consistent with a linear increase in $[\mathrm{La} / \mathrm{Fe}]$ with $[\mathrm{Fe} / \mathrm{H}]$ for $[\mathrm{Fe} / \mathrm{H}] \leq-1.3$, and the same slope of $1.7 \mathrm{dex} / \mathrm{dex}$ as for the Norris \& Da Costa (1995b) [Y, Ba, $\mathrm{Nd} / \mathrm{Fe}$ ] data, together with a constant value of $[\mathrm{La} / \mathrm{Fe}]$ above that $[\mathrm{Fe} / \mathrm{H}]$ value. The data presented in Johnson \& Pilachowski (2010) are also, at least qualitatively, consistent with this interpretation.

In the right panels of Figure 6 we show the same $s$-process-element-to-iron abundance ratios as a function of $[\mathrm{Fe} / \mathrm{H}]$ using the M22 data of Marino et al. (2009). In that data set abundance ratios are given from both the VLT/UVES and VLT/GIRAFFE samples for [Y/Fe] and $[\mathrm{Ba} / \mathrm{Fe}]$ (21 stars) while the data for $[\mathrm{Nd} / \mathrm{Fe}]$ come from the VLT/GIRAFFE observations only (13 stars, Marino et al. 2009). The M22 data show a distinct degree of similarity with those for $\omega$ Cen: again there is a steep rise in the $[s$-process $/ \mathrm{Fe}]$ element ratios with increasing $[\mathrm{Fe} / \mathrm{H}]$. The total range in $[s-$ process $/ \mathrm{Fe}]$ is $\sim 0.7 \mathrm{dex}$, slightly smaller than that seen in $\omega$ Cen. M22 also does not show the 'flat' part of the relation as it lacks stars of higher $[\mathrm{Fe} / \mathrm{H}]$ when compared to $\omega$ Cen.

To further investigate this similarity, we have endeavoured to transpose the $([s$-process/Fe $],[\mathrm{Fe} / \mathrm{H}])$ relations found for $\omega$ Cen to M22. The results of this are shown as the dot-dash lines in the right panels of Figure 6. For $[\mathrm{Y} / \mathrm{Fe}]$ and $[\mathrm{Ba} / \mathrm{Fe}]$, the dot-dash lines shown for M22 are exactly those for $\omega$ Cen save only that they have been shifted to lower $[\mathrm{Fe} / \mathrm{H}]$ values by $0.2 \mathrm{dex}$. In other words, a given $[\mathrm{Y}, \mathrm{Ba} / \mathrm{Fe}]$ value occurs at a $[\mathrm{Fe} / \mathrm{H}]$ value in $\mathrm{M} 22$ that is 0.2 dex lower than in $\omega$ Cen. For $[\mathrm{Nd} / \mathrm{Fe}]$, there are two dot-dash lines shown in the M22 panel. The first, which has lower $[\mathrm{Nd} / \mathrm{Fe}]$ at fixed $[\mathrm{Fe} / \mathrm{H}]$, is the same case as for $[\mathrm{Y} / \mathrm{Fe}]$ and $[\mathrm{Ba} / \mathrm{Fe}]$, i.e., the $\omega$ Cen relation shifted to lower $[\mathrm{Fe} / \mathrm{H}]$ values by $0.2 \mathrm{dex}$. Clearly it is a poor representation of the M22 $[\mathrm{Nd} / \mathrm{Fe}]$ data. The second is identical to the first except that it has been shifted vertically by $0.27 \mathrm{dex}$ in $[\mathrm{Nd} / \mathrm{Fe}]$ to match the $\mathrm{M} 22$

\footnotetext{
${ }^{4}$ We note that while mass transfer in binaries involving a thermally pulsing AGB star is often invoked to explain large $s$-process element enhancements for, e.g., halo field Ba II stars and specifically by Johnson et al. (2009) for the stars in $\omega$ Cen with high [La/Fe], the work of Mayor et al. $(1996,1997)$ shows that in $\omega$ Cen, the stars with large $s$-process enhancements appear generally to be single, unlike what is found in the halo field. In particular, while the multiple radial velocity observations of Mayor et al. $(1996,1997)$ have demonstrated that the $\omega$ Cen CH stars ROA 55 and ROA 77 are indeed long-period binaries, the same data set shows that the stars ROA 421 and ROA 451 (stars 51132 and 39048 in Johnson et al. (2009)), which have very large [La/Fe] values (Johnson et al. 2009) and which are classified as Ba II stars by Lloyd-Evans (1986), are apparently single stars. Hence adopting a "mass transfer in binary system' explanation for the $\omega$ Cen red giants with large $s$-process element enhancements may require that the binary is subsequently disrupted in the cluster environment. Johnson \& Pilachowski (2010), however, note that a proper accounting for hyperfine structure has revised downwards the $[\mathrm{La} / \mathrm{Fe}]$ values of Johnson et al. (2009), especially for the most La-rich stars. This reduces the need for invoking mass transfer in binary systems.
}

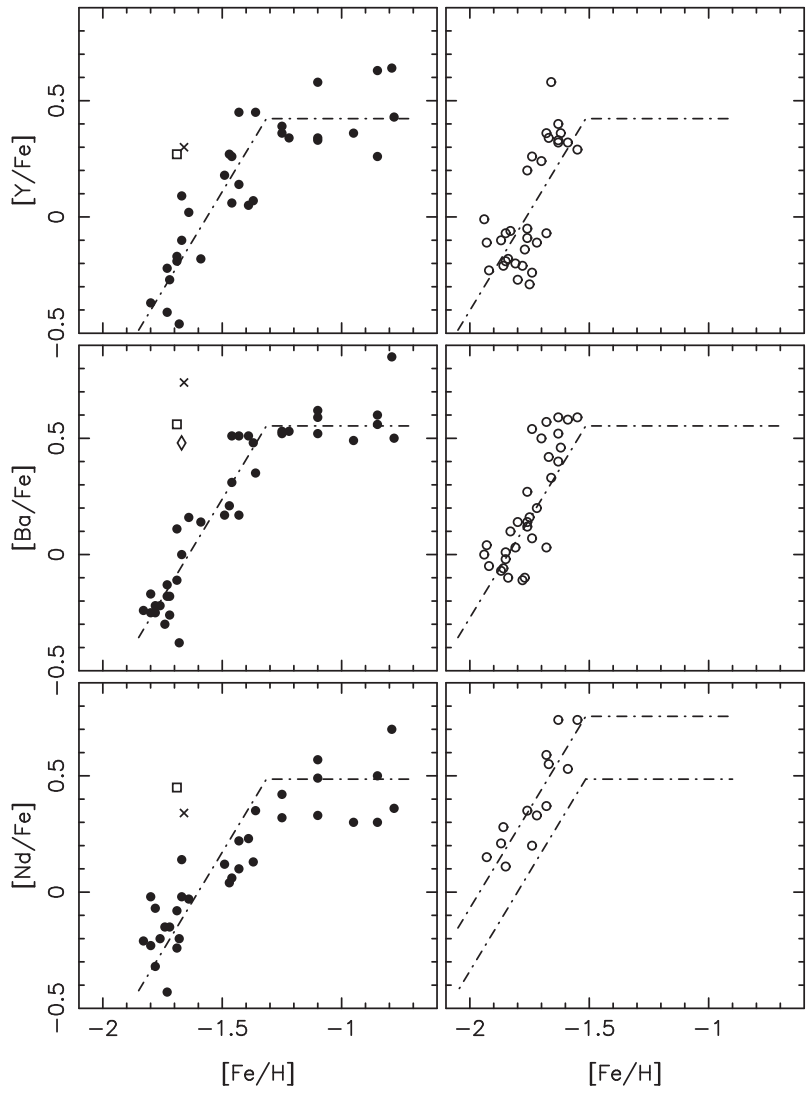

Figure 6 Element-to-iron abundance ratios for the $s$-process elements yttrium (upper panels), barium (middle panels) and neodymium (lower panels) as a function of $[\mathrm{Fe} / \mathrm{H}]$ for $\omega$ Cen red giants (left panels) and for M22 red giants (right panels). The dot-dash lines represent the relations adopted for the $\omega$ Cen stars; the slope of the linear portion is the same for all three element ratios. The open square is the $\mathrm{CH}$ star ROA 279 while the $\times$ sign is the CN-strong star ROA 144 (Norris \& Da Costa 1995b). These stars have been excluded from the fits, as was the $[\mathrm{Ba} / \mathrm{Fe}]$ data for ROA 53, shown as the open diamond in the middle left panel. In the right panels the $\omega$ Cen relations have been translated to the M22 stars as described in the text.

observations. This systematic relative offset in the $[\mathrm{Nd} / \mathrm{Fe}]$ versus $[\mathrm{Fe} / \mathrm{H}]$ relation between $\mathrm{M} 22$ and $\omega$ Cen may well have its origin in the $\mathrm{Nd}$ lines measured, and $g f$-values adopted for those lines, rather than any true difference in $[\mathrm{Nd} / \mathrm{Fe}]$ at equivalent $[\mathrm{Fe} / \mathrm{H}]$ values. In particular, we note that the $[\mathrm{Nd} / \mathrm{Fe}]$ values for M22 come from a single $\mathrm{Nd}$ II line at $532 \mathrm{~nm}$ (Marino et al. 2009) while those for $\omega$ Cen come from 3 to $6 \mathrm{Nd}$ II lines, including the $532 \mathrm{~nm}$ line (Norris et al. 1996a).

Intriguingly, the offset in $[\mathrm{Fe} / \mathrm{H}]$ required to match the $\omega$ Cen and M22 $[s / \mathrm{Fe}]$ relations is very similar to the difference between the peaks of the metallicity distribution functions for the two clusters, which is of order 0.1 dex (see Figure 1). Indeed, when we consider the uncertainties in the zero points of the abundance scales involved, the $\sim 0.2$ dex offset used in Figure 6 is fully consistent with the $\sim 0.1$ dex difference between the abundance distribution peaks. For example, we note that the M22 data in Figure 1 come from Da Costa et al. (2009) who measured $\mathrm{Ca}$ triplet line strengths calibrated to the $[\mathrm{Fe} / \mathrm{H}]$ scale of Kraft \& Ivans (2003). The $\omega$ Cen data, 
on the other hand, come from Norris et al. (1996) who measured $\mathrm{Ca}$ triplet and $\mathrm{Ca} \mathrm{K}$ line strengths and calibrated principally to the $[\mathrm{Ca} / \mathrm{H}]$ values of Norris \& Da Costa (1995b). A mean $[\mathrm{Ca} / \mathrm{Fe}]$ value was then applied to generate the $[\mathrm{Fe} / \mathrm{H}]$ distribution. Given these different approaches, it is indeed likely that the difference in abundance distribution peaks seen in Figure 1 is uncertain at the $\sim 0.1$ dex level. We note further that even with the most recent work, the absolute scale of globular cluster abundances remains uncertain at the $\sim 0.1$ dex level. For example, see the discussion of the various abundance scales in Carretta et al. (2009b).

Nevertheless, the similarity in the slopes of the $([s / \mathrm{Fe}]$, $[\mathrm{Fe} / \mathrm{H}])$ relations, and the similar overall range in $[s / \mathrm{Fe}]$ values, argue rather strongly that the $s$-process nucleosynthesis involved was very similar in both M22 and $\omega$ Cen. Further, it is worth noting that the slope for yttrium, which is a first $s$-process peak (or light-s) element, is evidently the same as for the second $s$-process peak (or heavy-s) elements $\mathrm{Ba}$ and $\mathrm{Nd}$ (and $\mathrm{La}$ ). Moreover, the abundance ratio $[\mathrm{Ba} / \mathrm{Y}]$, which is an [heavy-s/light-s] indicator, shows no dependence on $[\mathrm{Fe} / \mathrm{H}]$ in either $\omega$ Cen or M22, and takes the same value, $0.18 \pm 0.04$ (standard error of the mean) in both clusters, again emphasising the similarity of the $s$-process nucleosynthesis in these systems. This lack of any dependence on $[\mathrm{Fe} / \mathrm{H}]$ of the $[\mathrm{Ba} / \mathrm{Y}]$ ratio, despite the substantial changes in both $[\mathrm{Y} / \mathrm{Fe}]$ and $[\mathrm{Ba} / \mathrm{Fe}]$ with the $[\mathrm{Fe} / \mathrm{H}]$, shows that the mechanism contributing the $s$-process elements changed only as the regards the amount of $s$-process elements produced, and not in any other significant way, as the iron abundance increased.

The mechanism for generating $s$-process elements is most likely relatively low-mass thermally pulsing AGB stars (e.g., Busso et al. 1999). It appears then that there was an epoch in both $\omega$ Cen and M22 when the production of $s$-process elements exceeded the ongoing production of iron so that the $[s / \mathrm{Fe}]$ ratios increased substantially from low (pre-cluster $r$-process?) values to significantly in excess of the solar ratios. In M22 it would appear that the star formation then ceased while in $\omega$ Cen it continued, but without the excess $s$-process element production, as the $[s / \mathrm{Fe}]$ values now remain constant as $[\mathrm{Fe} / \mathrm{H}]$ rises.

\section{Discussion}

The nucleosynthetic processes that are likely to have occurred in $\omega$ Cen are outlined in detail in, for example, Johnson et al. (2009), Johnson \& Pilachowski (2010) and Romano et al. (2007, 2010), and need not be discussed further here. Rather the emphasis in this contribution is on the significant degree of similarity between $\omega$ Cen and M22 in their element-to-iron ratios as a function of $[\mathrm{Fe} / \mathrm{H}]$. This similarity, particularly for the $s$-process elements, suggests strongly that similar chemical enrichment processes occurred in both systems. As a result, any explanation for the chemical abundances, such as an origin in the nucleus of a now-disrupted dwarf galaxy, must apply to both if postulated for one. Consequently, the difference in mass between M22 and $\omega$ Cen (and for that matter also with M54, which has a comparable luminosity to $\omega$ Cen) is intriguing. Total mass is an important parameter for retaining gas from which to form successive generations of stars and so it is worthwhile to ask if it is possible that M22 could have been significantly more massive in the past. To investigate this question we assume that the M22 dwarf galaxy progenitor was disrupted at early times, as is the case for the $\omega$ Cen dwarf galaxy progenitor in the models of Bekki \& Freeman (2003). Thus we can compare the subsequent dynamical evolution of M22 with that of halo globular clusters. Under this assumption, it does not seem likely that M22 could have lost a substantial amount of stellar mass.

For example, in the compilation of Dinescu et al. (1999), the orbit of M22 is typical for inner halo objects (e.g., Carollo et al. 2007). It is prograde with $\Theta=178 \pm$ $20 \mathrm{~km} \mathrm{~s}^{-1}$, apo- and pericentric distances of approximately 9.5 and $2.9 \mathrm{kpc}$, respectively, and a period of 200 Myr (Dinescu et al. 1999). This suggests that M22 is unlikely to be strongly affected dynamically by disk and bulge shocks. Gnedin \& Ostriker (1997) reach similar conclusions, listing for M22 a destruction rate from disk and bulge shocks of $\sim 0.3$ inverse Hubble times, i.e., a destruction timescale exceeding 3 Hubble times. Further, with a half-mass two-body relaxation time of order 1.4 Gyr (Harris 1996; Gnedin \& Ostriker 1997), M22 is also unlikely to have lost significant mass through the evaporation of stars. Indeed the parameters for M22 place it inside the 'survival triangles' or 'vital diagrams' shown in Gnedin \& Ostriker (1997). Thus it seems improbable that M22 could have lost the factor of 5 or more by which its present day mass differs from that of $\omega$ Cen.

In this context it is then interesting to speculate that perhaps the M22 dwarf galaxy progenitor was of lower total mass than that postulated for the $\omega$ Cen system and that of the Sagittarius dwarf which currently has M54 as its central star cluster. This lower total mass for the original M22 system might then have meant that it did not retain gas in the central regions as efficiently, given the shallower potential well. The possible consequences could then be less mass built up in the central star cluster and insufficient time to allow the star formation and chemical evolution to evolve to the higher $[\mathrm{Fe} / \mathrm{H}]$ values seen in $\omega$ Cen. Similarly, in the scenario advanced for the ultimate fate of M54 (e.g., Carretta et al. 2010b), the M22 progenitor would not have had sufficient mass to acquire metal-rich stars from the nucleus of the dwarf galaxy in the tidal disruption process.

We note for completeness that Terzan 5, the other globular cluster with a significant internal $[\mathrm{Fe} / \mathrm{H}]$ abundance range (Ferraro et al. 2009), has recently been shown (Lanzoni et al. 2010) to be more massive than previously thought: Lanzoni et al. (2010) estimate the mass as $2 \times 10^{6}$ solar masses. This is comparable to that of $\omega$ Cen for which mass estimates range up to $5 \times 10^{6} M_{\text {sun }}$ (Meylan et al. 1995, see also van de Ven et al. (2006)). 
Further, as a metal-rich object in the Galactic Bulge, Terzan 5 is likely to be strongly affected by tidal shocks and may therefore have been yet more massive in the past.

One final question that the data presented here allow us to ask is whether there is any relation between the process(es) that govern the $\mathrm{O}-\mathrm{Na}$ anti-correlation and those that govern the $s$-process element enhancements. The former is seen in $\omega$ Cen and M22 (Norris \& Da Costa 1995a; Marino et al. 2009), and essentially all globular clusters (e.g., Carretta et al. 2010a), while the latter is seen in $\omega$ Cen and M22 as well as in a very small number of other clusters. For example, Yong et al. (2005) and Yong \& Grundahl (2008) have shown that there are small intrinsic variations in $s$-process element-to-iron abundance ratios in the clusters NGC $6752(\Delta[\mathrm{Y}, \mathrm{Zr}, \mathrm{Ba} / \mathrm{Fe}] \approx$ 0.1 dex $)$ and NGC $1851(\Delta[\mathrm{Zr}, \mathrm{La} / \mathrm{Fe}] \approx 0.3 \mathrm{dex})$. These variations appear to correlate with $[\mathrm{Na} / \mathrm{Fe}]$ and $[\mathrm{Al} / \mathrm{Fe}]$. No $[\mathrm{Fe} / \mathrm{H}]$ variations, however, were detected. Nevertheless, Carretta et al. (2010d) have recently presented evidence for the existence of two different groups of stars in NGC 1851 whose iron abundances differ by $0.06-0.08$ dex. Both components show the $\mathrm{O}-\mathrm{Na}$ anti-correlation and there is also a hint that the more metal-rich stars have slightly higher $[s / \mathrm{Fe}]$ values (Carretta et al. 2010d). A larger sample of NGC 1851 stars is required to establish whether this is a real effect and if the slope of any $([s / \mathrm{Fe}],[\mathrm{Fe} / \mathrm{H}])$ relation is similar to that seen in $\omega$ Cen and M22.

A relation between the $\mathrm{O}-\mathrm{Na}$ and $s$-process effects might be expected given that thermally pulsing AGB stars are the main source of $s$-process elements (e.g., Busso et al. 1999), and such stars, albeit of somewhat higher mass, are also often invoked to explain the O-Na anticorrelation (e.g., D'Antona \& Ventura 2007). The relatively large range in $[s / \mathrm{Fe}]$ seen in both $\omega$ Cen and M22 thus makes them ideal objects with which to investigate this question.

In Figure 7 we show the $[\mathrm{O} / \mathrm{Fe}],[\mathrm{Na} / \mathrm{Fe}]$ and $[\mathrm{Al} / \mathrm{Fe}]$ abundance ratios as a function of $[\mathrm{Ba} / \mathrm{Fe}]$ for $\omega$ Cen (left panels) and M22 (right panels) red giants. For $\omega$ Cen, we have plotted only those stars with $[\mathrm{Fe} / \mathrm{H}] \leq-1.3$, since below this value the abundance ratio changes significantly while above it the $[\mathrm{Ba} / \mathrm{Fe}]$ ratio is essentially constant (see Figure 6). Stars over the entire observed $[\mathrm{Fe} / \mathrm{H}]$ range are shown for M22. We note for completeness, however, that while the majority of the $\omega$ Cen metalrich stars are O-poor and Na, Al-rich, as seen in Figure 4, there do exist some metal-rich stars that are O-rich and $\mathrm{Na}, \mathrm{Al}-$ poor. Thus there is a range in $[\mathrm{O} / \mathrm{Fe}],[\mathrm{Na} / \mathrm{Fe}]$, and $[\mathrm{Al} / \mathrm{Fe}]$ at the essentially fixed (but high) $[\mathrm{Ba} / \mathrm{Fe}]$ value these stars possess.

Both the right and left panels of Figure 7 do not present any compelling case for a correlation between the $[\mathrm{O} / \mathrm{Fe}]$, $[\mathrm{Na} / \mathrm{Fe}]$, and $[\mathrm{Al} / \mathrm{Fe}]$ abundance ratios with $[\mathrm{Ba} / \mathrm{Fe}]$, in line with the conclusions of Smith (2008). At best there is a suggestion of a trend in which $[\mathrm{Na} / \mathrm{Fe}]$ might be slightly higher at larger $[\mathrm{Ba} / \mathrm{Fe}]$ values, though the $[\mathrm{O} / \mathrm{Fe}]$ and $[\mathrm{Al} / \mathrm{Fe}]$ values do not show any comparable trend. Plots of $[\mathrm{Na} / \mathrm{Fe}]$ and $[\mathrm{Al} / \mathrm{Fe}]$ against $[\mathrm{La} / \mathrm{Fe}]$ for $\omega$ Cen red giants using the data of Johnson et al. (2009) show the same result - there is some indication of a trend for increasing $[\mathrm{Na} / \mathrm{Fe}]$ with increasing $[\mathrm{La} / \mathrm{Fe}]$ but the $[\mathrm{Al} / \mathrm{Fe}]$ data are consistent with no correlation with $[\mathrm{La} / \mathrm{Fe}]$. The results of Johnson \& Pilachowski (2010) appear similar.

Thus is seems apparent that the process(es) which generate the $\mathrm{O}-\mathrm{Na}$ anti-correlation must be relatively distinct from those that generate the $s$-process element enhancements. This is despite the fact that in both $\omega$ Cen and M22 the existence of a range in $[\mathrm{Na} / \mathrm{Fe}]$ at fixed $[\mathrm{Ba} / \mathrm{Fe}]$, and vice versa, suggests that both processes were occurring together. It is hard to see how this can be the case invoking only AGB stars of different mass in a closed system - it is more likely that gas flows into and out of the star-forming systems are required as suggested by, for example, Bekki \& Norris (2006) and Romano et al. (2010). A detailed comparison of the abundance patterns in the Sagittarius dwarf galaxy central star cluster M54 with those of $\omega$ Cen and M22 would certainly assist in investigating this possibility.

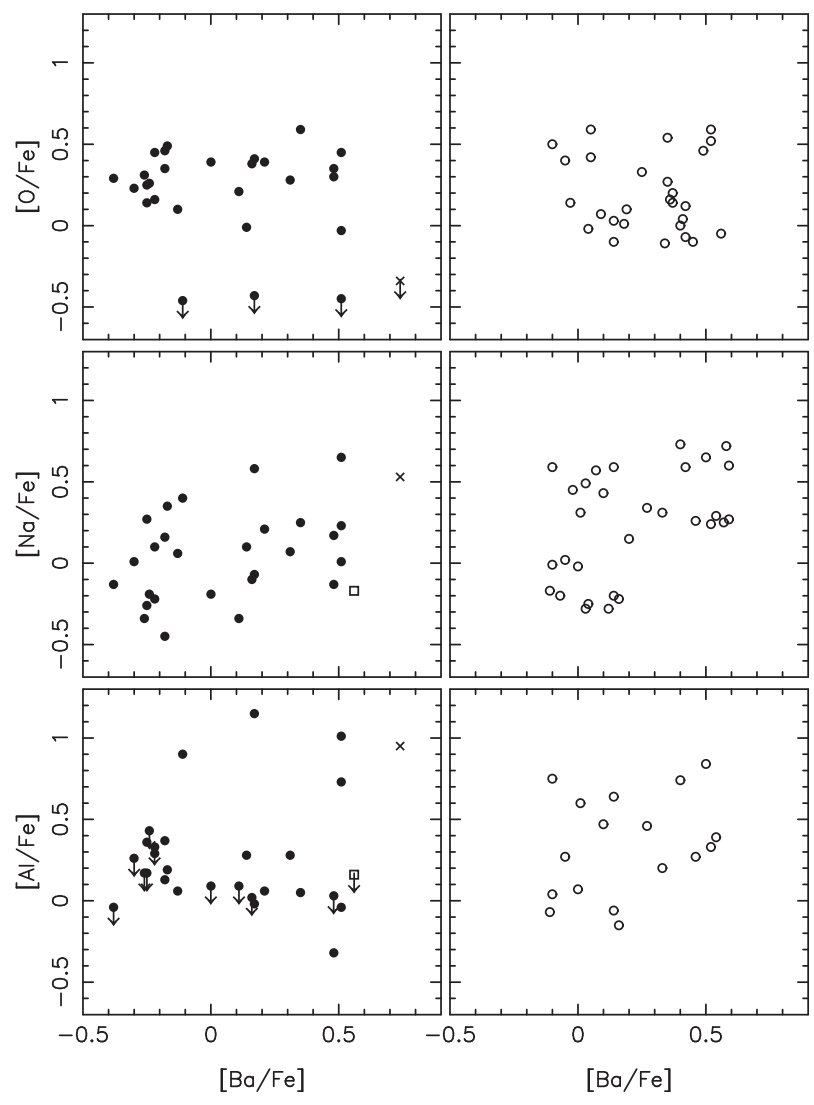

Figure 7 Oxygen-to-iron (upper panels), sodium-to-iron (middle panels) and aluminium-to-iron (lower panels) abundance ratios as a function of $[\mathrm{Ba} / \mathrm{Fe}]$ for red giants in $\omega$ Cen (left panels) and M22 (right panels). The $\omega$ Cen red giants shown all have $[\mathrm{Fe} / \mathrm{H}] \leq-1.3$ dex, the $[\mathrm{Fe} / \mathrm{H}]$ range over which the $[\mathrm{Ba} / \mathrm{Fe}]$ ratio changes significantly (see Figure 6). As in Figure 6, the open square in the $\omega$ Cen panels is the $\mathrm{C}-\mathrm{H}$ star ROA 279 while the $\times$ sign is the $\mathrm{CN}-$ strong star ROA 144. The lack of any correlation between these abundance ratios suggests that in both stellar systems the nucleosynthesis process that generates the $s$-process element enrichment is distinct from that which involves the $\mathrm{O}-\mathrm{Na}$ anti-correlation. 


\section{Acknowledgments}

The authors are grateful to Prof John Norris and Dr David Yong for their input to the original version of the manuscript and to the referee for their comments.

\section{References}

Anderson, J., Piotto, G., King, I. R., Bedin, L. R. \& Guhathakurta, P., 2009, ApJ, 697, L58

Bedin, L. R., Piotto, G., Anderson, J., Cassisi, S., King, I. R., Momany, Y. \& Carraro, G., 2004, ApJ, 605, L125

Bekki, K. \& Freeman, K. C., 2003, MNRAS, 346, L11

Bekki, K. \& Norris, J. E., 2006, ApJ, 637, 109

Bellazzini, M., Ibata, R. A., Chapman, S. C., Mackey, A. D., Monaco, L., Irwin, M. J., Martin, N. F., Lewis, G. F. \& Dalessandro, E., 2008, AJ, 136, 1147

Bellini, A., Bedin, L. R., Piotto, G., Milone, A. P., Marino, A. F. \& Villanova, S., 2010, AJ, 140, 631

Busso, M., Gallino, R. \& Wasserburg, G. J., 1999, ARA\&A, 37, 239

Cannon, R. D. \& Stobie, R. S., 1973, MNRAS, 162, 207

Carollo, D., Beers, T. C., Lee, Y. S., Chiba, M., Norris, J. E., Wilhelm, R., Sivarani, T., Marstellar, B., Munn, J. A., BailerJones, C. A. L., Fiorentin, P. R. \& York, D. G., 2007, Nature, 450, 1020

Carretta, E., Bragaglia, A., Gratton, R. G., Lucatello, S., Catanzaro, G., Leone, F., Bellazzini, M., Claudi, R., D’Orazi, V., Momany, Y., Ortolani, S., Pancino, E., Piotto, G., Recio-Blanco, A. \& Sabbi, E., 2009a, A\&A, 505, 117

Carretta, E., Bragaglia, A., Gratton, R., D’Orazi, V. \& Lucatello, S., 2009b, A\&A, 508, 695

Carretta, E., Bragaglia, A., Gratton, R. G., Recio-Blanco, A., Lucatello, S., D’Orazi, V. \& Cassisi, S., 2010a, A\&A, 516, 55

Carretta, E., Bragaglia, E., Gratton, R. G., Lucatello, S., Bellazzini, M., Catanzaro, G., Leone, F., Momany, Y., Piotto, G. \& D’Orazi, V., 2010b, ApJ, 714, L7

Carretta, E., Bragaglia, E., Gratton, R. G., Lucatello, S., Bellazzini, M., Catanzaro, G., Leone, F., Momany, Y., Piotto, G. \& D’Orazi, V., 2010c, A\&A, in press (arXiv:1006.5866v1)

Carretta, E., Gratton, R. G., Lucatello, S., Bragaglia, A., Catanzaro, G., Leone, F., Momany, Y., D’Orazi, V., Cassisi, S., D’Antona, F. \& Ortolani, S., 2010d, ApJ, in press (arXiv:1007.5301v1)

Da Costa, G. S., Held, E. V., Saviane, I. \& Gullieuszik, M., 2009, ApJ, 705, 1481

D'Antona, F. \& Ventura, P., 2007, MNRAS, 379, 1431

D'Antona, F., Bellazzini, M., Caloi, V., Fusi-Pecci, F., Galleti, S. \& Rood, R. T., 2005, ApJ, 631, 868

Decressin, T., Meynert, G., Charbonnel, C., Prantzos, N. \& Ekström, S., 2007, A\&A, 464, 1029

Denisenkov, P. A. \& Denisenkova, S. N., 1990, Sov. Astron. Lett., 16,275

Dinescu, D. I., Girard, T. M. \& van Altena, W. F., 1999, AJ, 117, 1792

Ferraro, F. R., Dalessandro, E., Mucciarelli, A., Beccari, G., Rich, R. M., Origlia, L., Lanzoni, B., Rood, R. T., Valenti, E., Bellazzini, M., Ransom, S. M. \& Cocozza, G., 2009, Nature, 462, 483

Freeman, K. C., 1993, The Globular Cluster - Galaxy Connection (ASP Conf. Ser. 48), Eds. Smith, G. H. \& Brodie, J. P. (San Francisco, CA: ASP), 608

Freeman, K. C. \& Rodgers, A. W., 1975, ApJ, 201, L71

Geyer, E. H., 1967, Z. fr Ap., 66, 16

Gnedin, O. Y. \& Ostriker, J. P., 1997, ApJ, 474, 223

Gratton, R., Sneden, C. \& Carretta, E., 2004, ARAA, 42, 385

Harris, W. E., 1996, AJ, 112, 1487

Ivans, I., Sneden, C., Kraft, R. P., Suntzeff, N. B., Smith, V. V., Langer, G. E. \& Fulbright, J. P., 1999, AJ, 118, 1273
Johnson, C. I., Pilachowski, C. A., Rich, M. R. \& Fulbright, J. P. 2009, ApJ, 698, 2048

Johnson, C. I. \& Pilachowski, C. A., 2010, ApJ, in press (arXiv: $1008.2231 \mathrm{v} 1)$

Kraft, R. P. \& Ivans, I. I., 2003, PASP, 115, 143

Langer, G. E., Hoffman, R. \& Sneden, C., 1993, PASP, 105, 301

Lanzoni, B., Ferraro, F. R., Dalessandro, E., Mucciarelli, A., Beccari, G., Miocchi, P., Bellazzini, M., Rich, R. M., Origlia, L., Valenti, E., Rood, R. T. \& Ransom, S. M., 2010, ApJ, 717,653

Lloyd-Evans, T., 1986, South Afr. Astron. Obs. Circ., 10, 1

Marino, A. F., Villanova, S., Piotto, G., Milone, A. P., Momany, Y., Bedin, L. R. \& Medling, A. M., 2008, A\&A, 490, 625

Marino, A. F., Milone, A. P., Piotto, G., Villanova, S., Bedin, L. R., Bellini, A. \& Renzini, A., 2009, A\&A, 505, 1099

Mayor, M., Duquennoy, A., Udry, S., Andersen, J. \& Nordstrom, B., 1996, The Origins, Evolutions, and Densities of Binary Stars in Clusters (ASP Conf. Ser. 90), Eds. Milone, E. F. \& Mermilliod, J.-C. (San Francisco, CA: ASP), 190

Mayor, M., Meylan, G., Udry, S., Duquennoy, A., Andersen, J., Nordstrom, B., Imbert, M., Maurice, E., Prevot, L., Ardeberg, A. \& Lindgren, H., 1997, AJ, 114, 1087

McWilliam, A., 1997, ARA\&A, 35, 503

Meylan, G., Mayor, M., Duquennoy, A. \& Dubath, P., 1995, A\&A, 303, 761

Milone, A. P., Piotto, G., King, I. R., Bedin, L. R., Anderson, J. Marino, A. F., Momany, Y., Malavolta, L. \& Villanova, S., 2010, ApJ, 709, 1183

Norris, J. E., 2004, ApJ, 612, L25

Norris, J. E. \& Da Costa, G. S., 1995a, ApJ, 441, L81

Norris, J. E. \& Da Costa, G. S., 1995b, ApJ, 447, 680

Norris, J. E., Da Costa, G. S. \& Tingay, S. J., 1996a, ApJS, 99, 637

Norris, J. \& Freeman, K. C., 1983, ApJ, 266, 130

Norris, J. E., Freeman, K. C. \& Mighell, K. J., 1996b, ApJ, 462, 241

Otsuki, K., Honda, S., Aoki, W., Kajino, T. \& Matthews, G. J., 2006, ApJ, 641, L117

Pancino, E., Pasquini, L., Hill, V., Ferraro, F. R. \& Bellazzini, M., 2002, ApJ, 568, L101

Piotto, G., 2009, The Ages of Stars, IAU Symposium 258, Eds Mamajek, E. E. Soderblom, D. R. \& Wyse, R. F. G. (Cambridge: CUP), 233

Piotto, G., Bedin, L. R., Anderson, J., King, I. R., Cassisi, S., Milone, A. P., Villanova, S., Pietrinferni, A. \& Renzini, A., 2007, ApJ 661, L53

Piotto, G., Villanova, S., Bedin, L. R., Gratton, R., Cassisi, S., Momany, Y., Recio-Blanco, A., Lucatello, S., Anderson, J., King, I. R., Pietrinferni, A. \& Carraro, G., 2005, ApJ, 621, 777 Renzini, A., 2008, MNRAS, 391, 354

Romano, D., Matteucci, F., Tosi, M., Pancino, E., Bellazzini, M., Ferraro, F. R., Limongi, M. \& Sollima, A., 2007, MNRAS, 376, 405

Romano, D., Tosi, M., Cignoni, M., Matteucci, F., Pancino, E. \& Bellazzini, M., 2010, MNRAS, 401, 2490

Sarajedini, A. \& Layden, A. C., 1995, AJ, 109, 1086

Smith, G. H., 2008, PASP, 120, 952

Smith, V. V., Suntzeff, N. B., Cunha, K., Gallino, R., Busso, M., Lambert, D. L. \& Straniero, O., 2000, AJ, 119, 1239

Sneden, C., Kraft, R. P., Shetrone, M. D., Smith, G. H., Langer, G. E. \& Prosser, C. F., 1997, AJ, 114, 1964

Ventura, P. \& D'Antona, F., 2008, MNRAS, 385, 2034

Woolley, R., 1966, Royal Obs. Ann., 2, 1

van de Ven, G., van den Bosch, R. C. E., Verolme, E. K. \& de Zeeuw, P. T., 2006, A\&A, 445, 513

Yong, D., Grundahl, F., Nissen, P. E., Jensen, H. R. \& Lambert, D. L., 2005, A\&A, 438, 875

Yong, D. \& Grundahl, F., 2008, ApJ, 672, L29 